\title{
Journey from oncologist to cancer survivor and patient advocate in the era of precision medicine
}

\author{
Burton M. Needles \\ Emeritus Staff, Mercy Hospital and Barnes-Jewish Hospital, Washington University School of Medicine, \\ St. Louis, Missouri 63110, USA
}

\begin{abstract}
In 2013, the American Association for Cancer Research (AACR) introduced the "Precision Medicine Series" of symposia. The goal of these conferences is to "highlight the incredible technology and advances in cancer research that together are enabling treatments that are precisely targeted to the unique molecular and genetic characteristics of an individual's cancer." This new series of AACR conferences reflects how patient treatment has evolved and continues to progress toward personalized treatments/medicine. It was in May of 2013 that I was diagnosed with a rare form of bladder cancer, plasmacytoid variant, for which survival statistics were grim, and the only genomic information available was the frequent somatic $\mathrm{CDH} 1$ loss-of-function mutation consistent with aggressive clinical behavior. The $\mathrm{CDH1}$ gene encodes for E-cadherin, which plays a role in cell-cell adhesions and acts as a tumor suppressor when expressed normally. This information was subsequently published, but not until April 2016 (Al-Ahmadie et al. 2017. Nat Genet 48: 356358). At the time, I was a practicing medical oncologist and, ironically, urologic cancers had been my area of interest, dating back to my fellowship at Memorial Sloan Kettering Cancer Center (MSKCC) in 1981. I decided to return to MSKCC for treatment based on their experience with rare urologic cancers as well as my own personal connection.
\end{abstract}

Corresponding author: burtonneedles@gmail.com

(c) 2018 Needles This article is distributed under the terms of the Creative Commons Attribution-NonCommercial License, which permits reuse and redistribution, except for commercial purposes, provided that the original author and source are credited.

Published by Cold Spring Harbor Laboratory Press

doi: $10.1101 /$ mcs.a002717
I recall my own personal frustration with my diagnosis because there were only a few case reports published, no standard therapy had shown benefit for this disease, and it was likely that my disease was not localized. Based on my oncologist's knowledge of how patients responded to individual drugs, a 4-mo course of a neoadjuvant three-drug combination chemotherapy was recommended followed by radical cystectomy. A pathologic complete response was achieved and this ultimately translated into a continuing 41/2-yr survivorship. Subsequently, an attempt was made to obtain a mutational profile of the somatic cancer genome, but because of technical issues and limited available specimen, it was unsuccessful. It was determined that there were no significant germline mutations in a constitutional sample.

My career in medical oncology encompassed a chemotherapy and immunotherapy approach that in 2013 was just beginning to incorporate the concept of integrating next-generational sequencing technology into clinical practice. Molecular testing had been established and included in treatment guidelines for lung, breast, colon, and melanoma. It took about 18 months for me to recover both physically and intellectually. I discovered that "chemo brain" was a real phenomenon which made it difficult to return to my oncology practice. Although I decided to retire from clinical practice, I still retained the desire to remain current in the field of oncology. Toward that end, I volunteered to be a Patient Advocate for the Molecular Tumor Board, as a part of the American Society of Clinical 
Oncology-sponsored Targeted Agent Profiling and Utilization Registry (TAPUR) study (ClinicalTrials.gov). This was based on my belief that as a clinician involved in clinical trials since 1982, and now as a cancer survivor, I could bring a unique perspective to this study.

The TAPUR study is designed for patients who had previously received (or stopped responding to) standard treatment, whose tumors had at least one genomic variation that could be targeted with specific drugs, or for whom no standard treatment existed. Patients whose tumors have a potentially actionable genomic variant are eligible to receive an FDA-approved, commercially available drug, regardless of tumor type.

The Molecular Tumor Board, which includes clinicians, researchers with experience in genomics, and patient advocates, is made available to treating physicians for consultation about proposed or alternate treatment options. A consensus interpretation is then provided. For me, participating in the Molecular Tumor Board presented an opportunity to see how sequencing of the cancer genome could be used to guide treatment options, and I was excited about continuing to be involved in clinical trial research. This trial was different from my previous clinical experience. It represented an acknowledgment of a transition to patientcentered clinical trial design for precision medicine with recommendations for individualized treatment. It also represented a new approach to clinical research. I had previous experience participating in clinical trials to gain regulatory drug approval for a specific tumor type, and data were evaluated from randomized controlled clinical trials including Phase II or large randomized Phase III studies. In the new era of precision medicine, the large "basket" trial (such as the TAPUR study) or "umbrella" trials were developed, which included data on the numerous genomic alterations and targeted therapy combinations available, and I was enthusiastic about participating in this new clinical trial design. It was evident that basket trials would enable enrollment of multiple tumor types and facilitate accrual of rare cancers (such as mine) and genomic alterations and potentially lead to more rapid approval of new therapies.

I have been very fortunate to be a $4 \frac{1}{2}-y$-y cancer survivor despite an ominous initial prognosis. It is now apparent that in addition to prospective trials, genomic testing may help us to understand clinical responses retrospectively based on patients who had exceptional responses to therapy (as in my case) and help guide drug development based on novel predictive biomarkers.

My experience as a medical oncologist tells me that although further studies of the role of precision medicine in oncology will be needed to help answer the question of whether precision medicine improves clinical outcomes, it is clear that we are transitioning to a more molecularly matched personalized therapy for patients. For rare and ultrarare tumors, for example, rather than relying on case reports and literature review, Kato et al. (2017) showed that identifying genome and protein markers for such patients was feasible, and when therapies were matched, $>50 \%$ of patients achieved stable disease $\geq 6 \mathrm{mo}$, partial response, or complete response. An acknowledgement of this approach of incorporating genomics into clinical decision-making is also exemplified by the FDA's approval earlier this year of pembrolizumab based on molecular profiling-marking the first tumor agnostic indication for a cancer drug (United States Food and Drug Administration).

\section{"...for successful implementation of genomics into clinical decision- making, organized molecular tumor boards are needed to support the community-based oncologist..."}

As a Patient Advocate and cancer survivor on the Molecular Tumor Board of the TAPUR study, I can appreciate the hope given to patients as well as the difficulties of the process matching them to a therapeutic option. For me, the opportunity to participate in the 
Competing Interest Statement

The author has declared no competing interest.
Journey from oncologist to cancer survivor

Molecular Tumor Board has been a remarkable educational experience. The therapeutic decision-making process for patients whose tumor may exhibit multiple actionable mutations can be challenging, and the evaluation and experience of the Molecular Tumor Board can be invaluable. This experience has also convinced me that for successful implementation of genomics into clinical decision-making, organized molecular tumor boards are needed to support the community-based oncologist, particularly in helping to understand the nuances of a molecular report and in clinical decision-making.

As I listen to the case history presentations, from a clinician's perspective I can appreciate the difficulties in deciding when to implement genomic testing in a patient's disease course. I know that further studies will be needed, and that the development of treatment algorithms will help answer this question. However, as a cancer survivor, the hope offered by the TAPUR study to patients as well as additional future investigations of targeted therapeutic options is a source for great optimism.

As a Patient Advocate and medical oncologist who was given a dismal prognosis on presentation, I am grateful that I have been able to witness and be a part of the emergence of a new era in cancer therapy.

\section{REFERENCES}

ClinicalTrials.gov https://clinicaltrials.gov/ct2/show/NCT02693535.

Kato S, Kurasaki K, lkeda S, Kurzrock R. 2017. Rare Tumor Clinic: the University of California San Diego Moores Cancer Center experience with a precision therapy approach. Oncologist 22: 1-8.

United States Food and Drug Administration. FDA grants accelerated approval to pembrolizumab for tissue/ site agnostic indication. US FDA web site. https://www.FDA.gov/drugs/information.drugs/approveddrugs/ ucm560040.htm. 


\title{
COLD SPRING HARBOR Molecular Case Studies
}

\section{Journey from oncologist to cancer survivor and patient advocate in the era of precision medicine}

\author{
Burton M. Needles
}

Cold Spring Harb Mol Case Stud 2018, 4: a002717

Access the most recent version at doi: $10.1101 /$ mcs.a002717

References This article cites 1 articles, 1 of which can be accessed free at: http://molecularcasestudies.cshlp.org/content/4/2/a002717.full.html\#ref-list-1

License This article is distributed under the terms of the Creative Commons Attribution-NonCommercial License, which permits reuse and redistribution, except for commercial purposes, provided that the original author and source are credited.

Email Alerting Receive free email alerts when new articles cite this article - sign up in the box at the Service top right corner of the article or click here. 